\title{
Impacto del uso de PCR múltiple en diagnóstico etiológico y tratamiento de infecciones respiratorias agudas en un hospital privado del norte del país
}

Diana G. Hernández-González, ${ }^{1}$ Lorena Rodríguez-Muñoz ${ }^{2 *}$ y Fortino Solórzano-Santos ${ }^{3}$

${ }^{1}$ Departamento de pregrado, Hospital Christus Muguerza, Saltillo, Coah.; ${ }^{2}$ Departamento de Infectología pediátrica, Hospital Christus Muguerza, Saltillo, Coah.; ' Unidad de Investigación en Enfermedades Infecciosas, Hospital Infantil de México Federico Gómez, Instituto Nacional de Salud, Ciudad de México. México

\section{Resumen}

Antecedentes y objetivo: Las enfermedades del sistema respiratorio son causa frecuente de prescripción de antibióticos. Actualmente se emplean nuevas tecnologías para su diagnóstico como el FilmArray Respiratory Panel. El objetivo de este estudio es identificar la correlación entre el diagnóstico y tratamiento de infecciones de vías respiratorias con el resultado de PCR para virus respiratorios. Material y métodos: Estudio descriptivo, transversal, retrospectivo, se incluyeron 134 pacientes atendidos en el Hospital Christus Muguerza en Saltillo, Coahuila. Para todos los casos se analizaron los resultados del panel y el tratamiento que recibieron los pacientes. Resultados: El 58 \% recibió tratamiento antibiótico a su ingreso, el $13 \%$ tratamiento combinado (antibiótico + antiviral), $27 \%$ recibió tratamiento sintomático y el $2 \%$ fue tratado con antiviral de primera instancia. Posterior al resultado el 38 \% continuó con antibiótico, el 30 \% con antibiótico y antiviral, 13.8 \% se manejó con antiviral y el $18.2 \%$ con tratamiento sintomático. Conclusión: $A$ pesar de la alerta mundial por la resistencia a los antimicrobianos se sigue tratando a los pacientes con antibióticos, por una situación que se cree está influenciada por varios factores.

PALABRAS CLAVE: Infecciones respiratorias. PR FilmArray. Antibióticos.

\section{Impact of the use of multiplex PCR on etiological diagnosis and treatment of acute respiratory infections in a private hospital of the north of the country}

\section{Abstract}

Background and objective: Respiratory system diseases represent one of the leading cause of prescription of antibiotics. At present, new technologies for the diagnosis are being used, including the FilmArray Respiratory Panel. The objective was to identify the correlation between the diagnosis and treatment of respiratory tract infections with the result of PCR for respiratory viruses. Material and methods: Descriptive, cross-sectional, restrospective study. 134 patients were included treated at the Christus Muguerza Hospital in Saltillo, Coahuila. For all cases, the positive results of this test and the treatment patients received were analyzed. Results: 58 \% received antibiotic treatment at admission, $13 \%$ received combined treatment (antibiotic + antiviral), $27 \%$ received symptomatic treatment since their admission and $2 \%$ whit antiviral. After receiving a positive result for respiratory viruses, $38 \%$ continued with antibiotics, $30 \%$ with antibiotics and antivirals, $13.8 \%$ only managed with

Correspondencia:

0016-3813/৫ 2020 Academia Nacional de Medicina de México, A.C. Publicado por Permanyer. Este es un artículo open access bajo la licencia
*Lorena Rodríguez-Muñoz

E-mail: dra.lorenardzm@gmail.com CC BY-NC-ND (http://creativecommons.org/licenses/by-nc-nd/4.0/).

Fecha de recepción: 04-06-2020

Fecha de aceptación: 27-08-2020 
antivirals and $18.2 \%$ with symptomatic treatment. Conclusion: Although we are currently on global alert for resistance to antibiotics, there is a lack of awareness about the prescription of antibiotics, due to a situation which is believed to be influenced by several factors.

KEY WORDS: Respiratory infections. PR FilmArray. Antibiotics.

\section{Introducción}

Las enfermedades del aparato respiratorio representan una de las primeras causas de atención médica en el mundo. Son padecimientos comunes en todas las edades, cuya etiología es variable dependiendo de la edad, las circunstancias medioambientales, el clima, el ámbito asistencial y las enfermedades de base. ${ }^{1,2}$ La mayoría de las infecciones respiratorias solo afectan al tracto respiratorio superior y pueden ser consideradas leves, de curso benigno y autolimitado (catarro común, rinitis y faringoamigdalitis). Se considera que en México la infección aguda de vías respiratorias superiores es la primera causa de enfermedad y el primer motivo por el cual se busca atención médica. Los virus respiratorios son la causa principal hasta en un $70-90 \%$ de los casos. Se han documentado en la edad adulta el rinovirus, seguido del virus de la influenza $A$ y $B$, coronavirus y adenovirus; en los niños los más comunes son el virus sincitial respiratorio (VSR), el virus de la parainfluenza $1,2,3$, el de la influenza $A$ y $B$, adenovirus y rinovirus. En una proporción menor, entre el 15 y el $30 \%$ de los casos en niños y entre el 5 y el $20 \%$ en adultos, la etiología es bacteriana: Streptococcus pyogenes, Streptococcus pneumoniae, Mycoplasma pneumoniae, Neisseria meningitidis y Neisseria gonorrhoeae. ${ }^{3}$

De las enfermedades respiratorias, se estima que solo el $5 \%$ puede implicar al tracto respiratorio intermedio e inferior (bronquitis, bronquiolitis y neumonía); son potencialmente más graves $y$, en muchos casos, requieren el ingreso hospitalario. ${ }^{4}$ Las complicaciones de estas enfermedades en la edad adulta se relacionan con comorbilidades en el paciente y con la senectud. ${ }^{2,5,6}$ Se considera que las infecciones agudas del tracto respiratorio inferior junto con otras enfermedades pulmonares crónicas se encuentran entre las causas más comunes de enfermedad grave y muerte en todo el mundo.?

En años previos, identificar la etiología por virus era difícil metodológicamente, por lo que en pocos hospitales se investigaba. En los últimos años las técnicas para el diagnóstico de las infecciones de vías respiratorias ha avanzado, utilizando actualmente la detección de ácidos nucleicos del virus, con pruebas como la reacción en cadena de la polimerasa (PCR), con sensibilidad del 95 al $100 \%$ y especificidad del 99 al $100 \%{ }^{8}$ Debido a que es común que en estas infecciones estén involucrados más de un virus, fue necesario el diseño de métodos de PCR múltiple en los que simultáneamente se puedan identificar diferentes virus. ${ }^{9}$ Otras opciones son la PCR acoplada a inmunoanálisis óptico enzimático, que son sistemas de detección de productos de amplificación mediante sondas que se encuentran inmovilizadas en una superficie de diferente naturaleza química. ${ }^{2}$

La situación actual en cuanto al uso de los antibióticos, antivirales y otros antimicrobianos es cada vez más alarmante, si bien se sabe que la resistencia a los antimicrobianos es un fenómeno que aparece de forma natural con el tiempo, este proceso se ve acelerado por el uso inadecuado de antimicrobianos, así como por su prescripción excesiva. Se considera que hasta en la mitad de los pacientes el uso de antibióticos es innecesario o inapropiado. ${ }^{10-12}$

Se espera que cuando el médico pueda contar con estudios que le permitan identificar la posible etiología de la infección respiratoria que está tratando, hará un uso más racional de los antibióticos. En este estudio se evaluó la conducta terapéutica en un hospital privado cuando se les proporcionó un resultado positivo identificando virus respiratorios. Se describen los agentes virales más frecuentes y las patologías respiratorias que cursaron en quienes se les realizó la prueba.

\section{Material y métodos}

Se realizó un estudio descriptivo, transversal y retrospectivo. Se seleccionaron los pacientes de todas las edades que hubiesen tenido resultados positivos para virus respiratorios en una prueba de reacción de PCR multiplex realizada en el periodo de enero 2017 a marzo de 2019. Se incluyeron los pacientes que tuvieron expediente completo dentro del Hospital Christus Muguerza, un hospital privado de la ciudad de Saltillo, Coahuila. 
A todos los pacientes incluidos en el estudio se les realizó una prueba de PCR para virus respiratorio durante su estancia en el hospital. Se utilizó el FilmArray Respiratory Panel ${ }^{\circledR}(\mathrm{RP})$, que es una prueba cualitativa para la detección e identificación simultánea de múltiples ácidos nucleicos de virus y bacterias en aspirado nasofaríngeo. A cada paciente se le tomó un volumen mínimo de muestra de $0.3 \mathrm{ml}(300 \mu \mathrm{l}))$ del tracto respiratorio. Es una prueba que identifica en forma simultánea los siguientes virus y bacterias: adenovirus, coronavirus 229E, coronavirus HKU1, coronavirus NL63, coronavirus OC43, virus de la influenza A (con subtipado para los genes de la hemaglutinina $\mathrm{H} 1$, H1-2009 y H3) y la influenza B, metapneumovirus humano, virus de la parainfluenza 1, parainfluenza 2, parainfluenza 3 y parainfluenza 4 , virus sincicial respiratorio, rinovirus/enterovirus, Bordetella pertussis, Chlamydophila pneumoniae y Mycoplasma pneumoniae.

La muestra fue introducida en el cartucho FilmArray $\mathrm{RP}$, se colocó el cartucho en el instrumento/Module FilmArray e inició la prueba; se generó automáticamente un informe de la prueba al final del análisis. El proceso completo tarda aproximadamente una hora. La prueba posee una sensibilidad y especificidad del 95 y el $99 \%$ respectivamente. $^{13}$

En cada caso positivo se analizaron: el diagnóstico clínico radiológico establecido por el médico tratante, el tratamiento prescrito, la conducta sobre el tratamiento que se siguió al tener el resultado de la prueba, el tiempo de prescripción de antimicrobianos y las condiciones al egreso del paciente.

\section{Resultados}

La muestra inicial fue de 172 pacientes de todas las edades, de los cuales se excluyeron 38, por no contar con un expediente completo.

La muestra final fue de 134 pacientes, el $49.2 \%$ fueron hombres y el $50.8 \%$ mujeres; se incluyeron pacientes de todas las edades y de ellos el $56 \%$ fueron lactantes, el $16 \%$ preescolares, el $2 \%$ escolares, el $4 \%$ adolescentes y el $22 \%$ adultos.

De los resultados del panel viral, en el $25 \%$ de las muestras se identificó VSR, seguido de influenza A (18\%), rinovirus/enterovirus (10\%), metapneumovirus (10\%), influenza B $(9 \%)$, parainfluenza (5\%), coronavirus (4\%), B. pertussis (2\%), M. pneumoniae $(1 \%)$ y en el $16 \%$ de las muestras se aisló más de un virus (Figura 1).
Los diagnósticos clínicos radiológicos en el total de pacientes fueron: neumonía un $42 \%$, el $10 \%$ con bronquiolitis, el $17 \%$ con influenza, el $8 \%$ con bronquitis, crisis asmática en un $2 \%$, rinofaringitis en un $2 \%$ y el $19 \%$ con otros diagnósticos.

Del $100 \%$ de pacientes el $58 \%$ recibió solo tratamiento antibiótico a su ingreso, el $13 \%$ recibió tratamiento combinado (antibiótico + antiviral), el $27 \%$ de los pacientes recibieron tratamiento sintomático y el $2 \%$ fue tratado de primera instancia con antiviral.

Al obtener el resultado del panel viral positivo, de los pacientes que recibieron inicialmente antibiótico (71\%) solo en el $10 \%$ se suspendió este tratamiento; el $2.3 \%$ continuaron solo con el antiviral que fue administrado desde su ingreso, en el $1.5 \%$ se suspendió el antibiótico y se agregó antiviral, y en el $6.2 \%$ se suspendió el antibiótico y fue tratado con tratamiento sintomático. El $22 \%$ agregaron antiviral al tratamiento antibiótico que ya tenían y el $8 \%$ continuaron con tratamiento combinado; el $31 \%$ continuaron con antibiótico únicamente. El $12 \%$ de los pacientes continuaron únicamente con tratamiento sintomático, el $7 \%$ agregaron antibiótico a su tratamiento sintomático posterior al resultado positivo del panel respiratorio y el $10 \%$ agregó antiviral al tratamiento sintomático.

Posterior al resultado positivo, el $38 \%$ recibió como tratamiento definitivo antibiótico, el $30 \%$ se trató con tratamiento combinado (antibiótico + antiviral), el $13.8 \%$ fue tratado solo con antiviral y el $18.2 \%$ con tratamiento sintomático (Figura 2).

Del $68 \%$ de pacientes que recibieron antibiótico de forma empírica o como tratamiento definitivo, el $41 \%$ recibieron más de un antibiótico durante su estancia hospitalaria. Como monoterapia o asociado a otro antibiótico el $45 \%$ recibió una cefalosporina, el $17 \%$ macrólidos, el $11 \%$ quinolonas y el $5 \%$ otros. En el $39 \%$ de los pacientes se encontraron diagnósticos o estudios de laboratorio (como cultivo de secreción bronquial) que justificaron el uso de antibióticos.

Los días de estancia intrahospitalaria en los pacientes que recibieron tratamiento antibiótico a pesar de obtenerse un panel viral positivo (38\%) se reportaron entre 5-6 días, al igual que el $30 \%$ que fue tratado con antibiótico y antiviral. En el $13.8 \%$ que recibió tratamiento antiviral la estancia varió de 3 a 4 días, en los que recibieron tratamiento sintomático su estancia hospitalaria varió de 4 a 5 días. Hubo dos defunciones (1.5\%), un paciente ameritó traslado a una unidad de alta especialidad y 131 no presentaron complicaciones y egresaron por mejoría. 


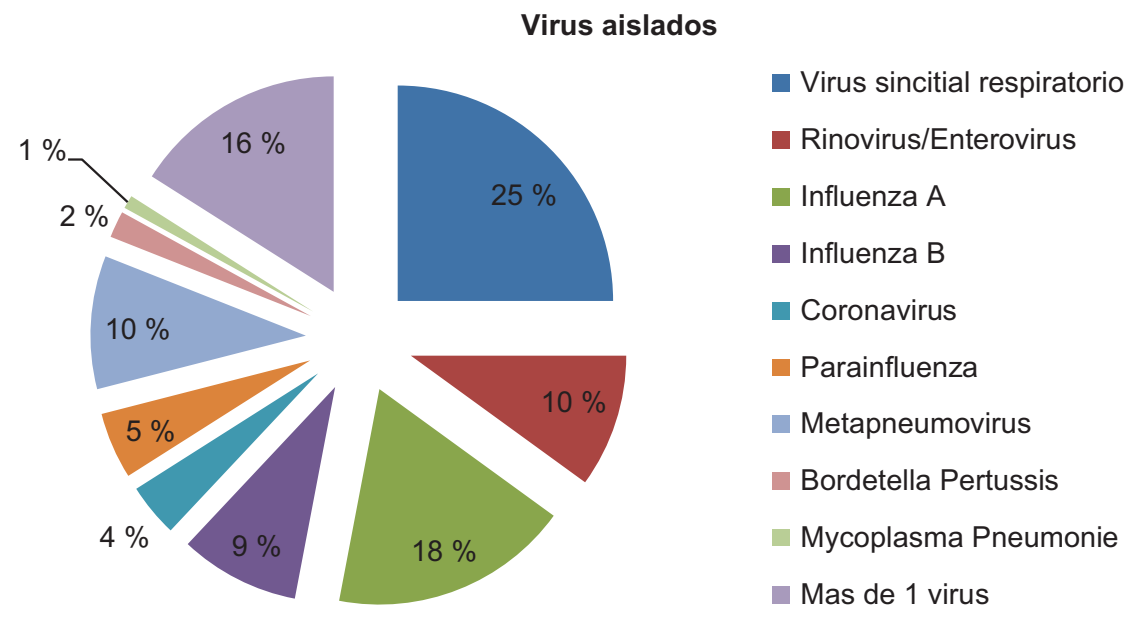

Figura 1. Distribución porcentual de virus identificados por reacción en cadena de la polimerasa en muestras nasofaríngeas.

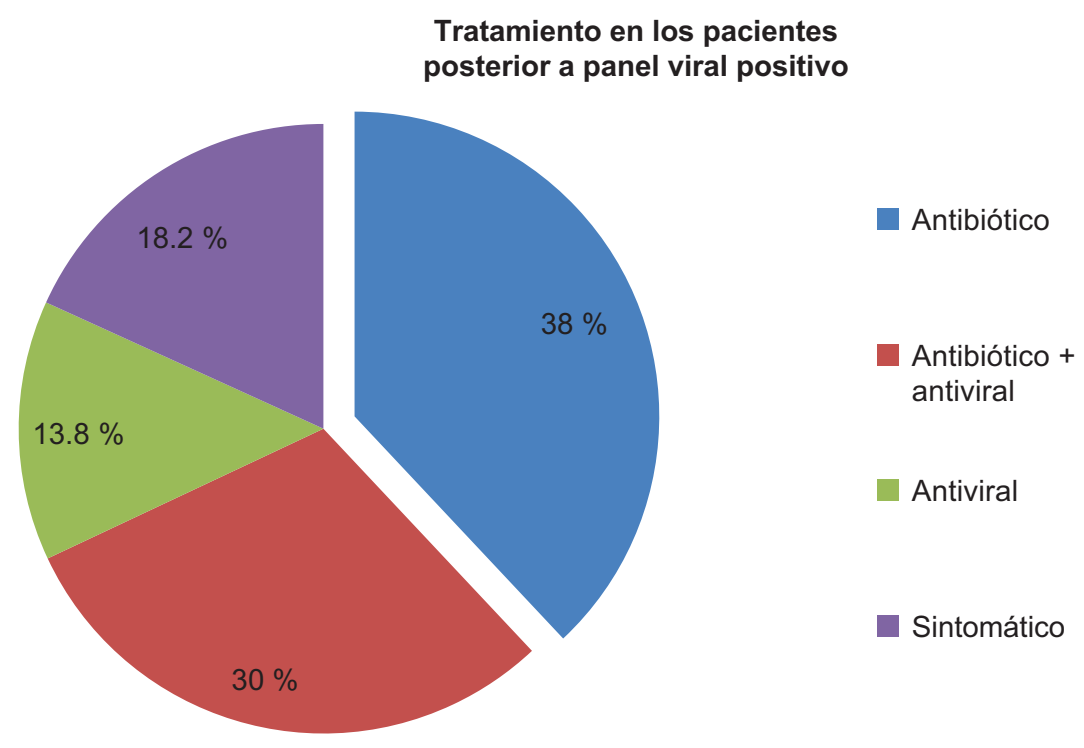

Figura 2. Tratamiento final recibido por los pacientes a pesar de tener un reporte para virus positivo.

\section{Discusión}

Existen diversos estudios que muestran tanto en pacientes adultos como pediátricos una alta prevalencia de agentes virales como causantes de los procesos agudos respiratorios que los llevan a la hospitalización. ${ }^{14,15}$

Conocer la etiología y el cuadro clínico de las enfermedades respiratorias, apoyados en los métodos actuales para su diagnóstico, debería facilitar la toma de decisiones sobre el tratamiento adecuado para cada paciente. Cuando el cuadro clínico mantiene una adecuada correlación con el hallazgo de laboratorio, el médico tiene los elementos para sustentar su decisión terapéutica. ${ }^{5}$

En este estudio se evaluó qué tanto influye un resultado positivo del panel respiratorio por PCR con el tratamiento que recibieron los pacientes.

A nivel mundial es variable la conducta de reducir el uso de antibióticos al tener un resultado positivo en un panel viral. En un estudio en Suecia de los pacientes en los que se identificó algún virus el $21 \%$ fue tratado con antibióticos de forma definitiva. ${ }^{9}$ En el presente estudio, el $71 \%$ de los pacientes recibieron algún antibiótico a su ingreso y el $68 \%$ lo recibió como 
tratamiento definitivo aun cuando posteriormente se tuvo un resultado positivo para virus. El resultado es similar a la experiencia en Sri Lanka ${ }^{16}$, en pacientes ambulatorios, en donde durante la época endémica de influenza realizando la detección del virus solo se pudo reducir el $20 \%$ del uso de antibióticos, del 83.7 al $62.3 \%$, a pesar de una prueba positiva para influenza; el alto volumen de pacientes y el miedo a la sobreinfección bacteriana fueron factores importantes en el uso excesivo de antibióticos en ese estudio.

El diseño en nuestro estudio no permite conocer los factores que influyeron en que los médicos tratantes continuaran con la prescripción de antibióticos; en el $39 \%$ de los expedientes se encontró alguna justificación para su uso por sobreinfección bacteriana, no se encontraron datos de mayor gravedad y quizá el factor que mas influyó fue el haber hospitalizado a los pacientes. Los antibióticos que más se utilizaron como tratamiento definitivo fueron cefalosporinas (45\%) y macrólidos (17\%).

En las muestras analizadas tuvimos un predominio del VSR en un $25 \%$, seguido de influenza $A$ con un $18 \%$ y $\mathrm{B}$ con un $9 \%$; en el $16 \%$ de las muestras se detectó más de un virus. En un estudio realizado en nuestro país en pacientes con enfermedad semejante a influenza la proporción de virus identificados fue con predominio de rinovirus (36.5\%), seguido de influenza (22.6\%), coronavirus (17.9\%) y VSR (14.2\%), aunque las muestras parecen corresponder a temporadas de influenza; de esta muestra el $47 \%$ fueron hospitalizados. ${ }^{17}$ Lo anterior refleja la amplia circulación de virus respiratorios, que pueden condicionar cuadro moderados a graves que requieren manejo hospitalario. Particularmente, el VSR y el virus de la influenza suelen producir cuadros más graves. En temporada invernal predominan los rinovirus, sin embargo, en los casos con neumonía comunitaria o con infección respiratoria que requiera hospitalización predominan el virus de la influenza, el VSR y el metapneumovirus. ${ }^{17,18}$ Existe discrepancia sobre si la presencia de coinfecciones virales puede causar un curso más grave de la infección. 17,18

A pesar de que actualmente la resistencia a los antimicrobianos es uno de los principales problemas de salud pública en el mundo ${ }^{11}$, continúa su prescripción no razonada. Se requiere darle el peso correcto a la asociación de estudios de laboratorio que detectan virus en pacientes con infecciones respiratorias moderadas a graves y poder aceptar que en el mayor porcentaje de esos casos no se requiere el uso de antimicrobianos. Hay estudios que claramente encuentran virus respiratorio en pacientes con neumonía comunitaria y pacientes con infecciones respiratorias graves que requieren manejo en terapia intensiva ${ }^{18,19}$ en los que no se demostró asociación bacteriana. El comportamiento médico en cuanto a su decisión terapéutica en un hospital privado muestra que a pesar de haber hecho un estudio para la búsqueda de virus como causantes de infección respiratoria aguda, no se utilizaron los resultados obtenidos para generar una modificación en cuanto al uso de antibiótico.

Se requerirá conocer cuáles son los motivos que originan que se continúe el manejo antimicrobiano aun en presencia de etiología viral. Probablemente no hay confianza en los estudios de laboratorio por desconocer su sensibilidad y especificidad, o existe temor de no ofrecer un tratamiento "que pueda curar la infección”, especialmente por haber pocas alternativas antivirales, y mayor presión familiar, porque en hospitales privados el médico tratante asume las decisiones y responsabilidades con su paciente.

Se requiere mayor difusión de la información sobre la participación de los virus en infecciones respiratorias de evolución moderada a grave que no requieren tratamiento antimicrobiano. Probablemente se requiere tiempo para aceptar la utilidad de las nuevas técnicas diagnósticas de laboratorio que recientemente se han incorporado a los hospitales.

\section{Conflicto de intereses}

Los autores declaran no tener conflicto de intereses alguno.

\section{Financiamiento}

Los autores no recibieron patrocinio para llevar a cabo este artículo.

\section{Responsabilidades éticas}

Protección de personas y animales. Los autores declaran que para esta investigación no se han realizado experimentos en seres humanos ni en animales.

Confidencialidad de los datos. Los autores declaran que han seguido los protocolos de su centro de trabajo sobre la publicación de datos de pacientes.

Derecho a la privacidad y consentimiento informado. Los autores declaran que en este artículo no aparecen datos de pacientes. 


\section{Bibliografía}

1. Lopardo G, Calmaggi A. Consenso sobre diagnóstico y tratamiento de infecciones de vías respiratorias altas. Medicina. 2012;72:484-94.

2. Eiros JM, Ortiz de LR. Diagnóstico microbiológico de las infecciones virales respiratorias. Enferm Infecc Microbiol Clin. 2009;27(3):168-77.

3. Diagnóstico y manejo de la infección aguda de vías aéreas superiores en pacientes mayores de 3 meses hasta 18 años de edad [Internet] Gobierno de México, Catálogo maestro, Guías de práctica clínica; 4 de septiembre de 2015 [fecha de actualización:L noviembre de 2016]. Disponible en: https://cenetec-difusion.com/gpc-sns/?p=1078

4. Información relevante: infecciones respiratorias agudas [Internet]. México: Subsecretaría de Prevención y Promoción de la Salud, Dirección General de Epidemiología (DGE), Sistema de Notificación Semanal de casos Nuevos [fecha de acceso: 23 de marzo de 2017]. Disponible en: https://www.gob.mx/cms/uploads/attachment/file/220600/IRA_2017 SE16.pdf

5. Guía de vigilancia epidemiológica y recomendaciones para la prevención y diagnóstico de las infecciones respiratorias agudas en Argentina [Internet]. Argentina: Ministerio de Salud; actualización 2018. Disponible en: http://www.entrerios.gov.ar/msalud/wp-content/uploads /2018 /06/20 18-07_guia-infecciones-respiratorias-agudas.pdf

6. Pneumonia [Internet]. American Lung Association; 2020. Disponible en: http://www.lung.org/lung-health-and-diseases/lung-disease-lookup/pneumonia/

7. Foro de las Sociedades Respiratorias Internacionales. El impacto gobal de la Enfermedad Respiratoria. Segunda edición. México: Asociación Latinoamericana de Tórax; 2017.

8. Bayona Y, Niederbacher J. Infecciones respiratorias virales en pediatría: generalidades sobre fisiopatogenia, diagnóstico y algunos desenlaces clínicos. Med UIS. 2015;28(1):133-41.

9. Rhedin S, Lindstrand A. Clinical utility of PCR for common viruses in acute respiratory illness. Pediatrics. 2014;133(3):538-45.
10. Infectious Diseases Society of America (IDSA). Antimicrobial resistance: a public health crisis. 2016. http://www.idsociety.org/topic_antimicrobial_resistance/\#

11. Resistencia a los antimicrobianos [Internet]. Organización Mundial de la Salud; 13 de octubre de 2020. Disponible en: https:/www.who.int/antimicrobial-resistance/es/

12. Hersh AL, Jackson MA. Principles of judicious antibiotic prescribing for upper respiratory tract infections in pediatrics. Pediatrics. 2013;132(6):1146-54.

13. BioFire Diagnostics. FilmArray Respiratory Panel (PR). Manual de instrucciones 2016. pp. 7-31

14. Yoshii Y, Shimizu K, Morozumi M, Chiba N, Ubukata K, Uruga $\mathrm{H}$, et al. Detection of pathogens by real-time PCR in adult patients with acute exacerbation of bronquial astma. BMC Pulm Med. 2017;17:150.

15. Jiang W, Wu M, Zhou J, Wang Y, Hao C, Ji W, et al. Etiologic spectrum and occurrence of coinfections in children hospitalized with community-acquired pneumonia. BMC Infect Dis. 2017;17:787.

16. Tillekeratne LG, Bodinayake CK, Nagahawatte A, Vidanagama D, Devasiri V, Arachchi WK, et al. Use of rapid influenza testing to reduce antibiotic prescriptions among outpatients with Influenza-Like illness in Southern Sri Lanka. Am J Trop Med Hyg. 2015; 93(5):1031-7.

17. Noyola DE, Hunsberger S, Valdés Salgado R, Powers JH 3rd, Galindo-Fraga A, Ortiz-Hernández AA, et al. Comparison of rates of hospitalization between single and dual virus detection in a Mexican cohort of children and adults with Influenza-Like illness. Open Forum Infect Dis. 2019:6(11):ofz424.

18. Rhedin S, Lindstrand A, Hjelmgren A, Ryd-Rinder M, Öhrmalm L, Tolfvenstam $\mathrm{T}$, et al. Respiratory viruses associated with community-acquired pneumonia in children: matched case-control study. Thorax. 2015;70(9):847-53.

19. Becerra M, Fiestas V, Tantaleán J, Mallma G, Alvarado M, Gutiérrez V, et al. Etiología viral de las infecciones respiratorias agudas graves en una unidad de cuidados intensivos pediátricos. Rev Peru Med Exp Salud Publica. 2019;36(2):231-8. 